\title{
Influence of Recurrent Electroconvulsive Therapy on Cardiac Function
}

\author{
Gokay Alpak $^{\mathrm{a}}$ Suleyman Ercan ${ }^{\mathrm{b}}$ Hayri Alicid $^{\mathrm{d}}$ Feridun Bulbul $^{\mathrm{a}}$ \\ Gokhan Altunbas $^{\mathrm{e}}$ Demet Alici $^{\mathrm{a}}$ Vahap Saricicek ${ }^{c}$ Vedat Davutoglu $^{\mathrm{b}}$ \\ Departments of a Psychiatry, ${ }^{b}$ Cardiology and ${ }^{\mathrm{C}}$ Anesthesiology, Gaziantep University, School of Medicine, and \\ dDepartment of Cardiology, 25 Aralik State Hospital, Gaziantep, and eDepartment of Cardiology, Kilis State Hospital, \\ Kilis, Turkey
}

\section{Key Words}

Echocardiography · Diastolic function - Electroconvulsive therapy

\begin{abstract}
Objective: To investigate the effects of recurrent electroconvulsive therapy (ECT) on cardiac function as assessed by echocardiography. Subjects and Methods: Twenty-three patients (11 males and 12 females) with different psychiatric disorders who were apparently free of any cardiovascular disorders and underwent ECT were enrolled in the study. Echocardiographic findings including diastolic mitral inflow and tissue Doppler features were recorded at baseline and at the end of the 7th and last ECT in all patients. Results: The mean age of the patients was $37.95 \pm 13.28$ years (range 1971). There was no significant difference in mitral $E$ wave velocities and tissue Doppler E' velocities between the baseline and after the 1 st ECT ( $p=0.161$ and $p=0.083$, respectively). The results were similar after the last ECT session $(p=0.463$ and $p=0.310$, respectively). However, there was a significant increase in transmitral A wave velocity after the $1 \mathrm{st}$ and 7 th ECT session compared to the values at baseline $(p=0.008$ and $p=0.017$, respectively). Conclusion: The mitral diastolic inflow A wave velocity increased $20 \mathrm{~min}$ after ECT, and this
\end{abstract}

increase persisted after recurrent ECT sessions. This finding could be considered as an indicator of acutely increased sympathetic tone.

(c) 2014 S. Karger AG, Basel

\section{Introduction}

Electroconvulsive therapy (ECT) induces widespread convulsions with the use of electrical stimuli. ECT is a primary or add-on therapy with medical therapy for various disorders including major depression, bipolar disorders, schizophrenia and catatonia. Except for increased intracranial pressure, there is no solid contraindication for ECT [1].

Recently, with the use of ECT increasing, various complications have been reported. Tachy-/bradyarrhythmias and hypertensive crises are seen more frequently than more serious complications such as myocardial infarction, pulmonary edema, cardiac rupture and asystole [1, 2]. These complications tend to occur especially in patients with a prior history of cardiovascular disease $[1,2]$; Kadoi et al. [3], however, have reported that ECT might actually trigger electrocardiographic abnormalities and systolic/diastolic dysfunction of the heart even in patients 
without any known cardiovascular disease. To our knowledge, there is no study in the literature that has investigated the effects of recurrent ECT on subjects free of any cardiovascular disease using tissue Doppler techniques. The aim of this study was to investigate the effect of recurrent ECT (7 times) on the various echocardiographic parameters of myocardial functions such as systolic and diastolic functions, mitral inflow properties and tissue Doppler parameters.

\section{Subjects and Methods}

Twenty-three patients ( 11 males and 12 females) who were admitted to the Gaziantep University School of Medicine between May and July 2012 with different psychiatric disorders and a recommendation for ECT as the initial treatment option were enrolled in this study. Approval of the local Institutional Ethics Committee was obtained before commencing the study and the patients gave their written informed consent. All patients underwent detailed echocardiographic examination and only those with a completely normal examination were deemed eligible for the study. Exclusion criteria were a prior history of cardiovascular disease (coronary artery disease, heart failure, myocardial infarction, stroke, transient ischemic attack or peripheral arterial disease), diabetes mellitus, hypertension, hyperlipidemia and chronic kidney disease. All patients received their usual chronic medications (such as antidepressants) after ECT and echocardiographic examinations. None received cardiac drugs such as beta-adrenoceptor antagonists and/or calcium-channel-blocking drugs. They all routinely received $0.1 \mathrm{mg} / \mathrm{kg}$ atropine sulfate $\mathrm{i} . \mathrm{m} .30 \mathrm{~min}$ prior to the ECT procedure. Systolic and diastolic blood pressure and heart rate were monitored at 2-min intervals before ECT until the end of the procedure. After the administration of propofol $(1 \mathrm{mg} / \mathrm{kg})$ over $10 \mathrm{~s}$ and loss of consciousness, succinylcholine $(1 \mathrm{mg} / \mathrm{kg})$ was administered as well as assisted mask ventilation with $100 \%$ oxygen. Within 2-3 min after the propofol injection, bilateral ECT was performed. For each ECT session, the initial charge was $504 \mathrm{mC}$.

\section{Transthoracic Echocardiographic Evaluation}

Detailed echocardiographic examination was performed before and $20 \mathrm{~min}$ after the 1st ECT. Repeat echocardiography was performed 20 min after the 7th ECT. Echocardiographic measurements were obtained using Vivid 7 Dimension (GE Vingmed Ultrasound AS, Horten, Norway) with a $2.5-\mathrm{MHz}$ transducer. Leftventricle (LV) end-diastolic and end-systolic diameters, interventricular septum and posterior wall thickness, LV ejection fraction and left atrial diameters were recorded in the parasternal longaxis view. Measurements of LV inflow properties were obtained from the apical 4-chamber view with a Doppler sample parallel to the LV long axis at the mitral annulus level. Mean values of the measurements were recorded. Early (E) and late (A) diastolic flow velocities and their ratios (E/A) were recorded [4]. Tissue Doppler imaging was used in the apical 4-chamber view and pulsed-wave Doppler recordings were obtained with a $5-\mathrm{cm}$ sampling width at the junction of the posterior wall and mitral annulus. The sampling volume was aligned parallel to the wall, and the peak early (E') and late $\left(\mathrm{A}^{\prime}\right)$ diastolic motion velocity was recorded [5]. All measure- ments were performed 3 times and mean values were recorded. S.E. and H.A. interpreted the echocardiograms and they were both blinded to the timing of the examination.

\section{Statistical Analysis}

Statistical analysis was performed using SPSS 16.0 and data were recorded as mean \pm standard deviation. Descriptive methods (i.e. mean and standard deviation) were used with the paired test for parameters with normal distribution. Results were evaluated with a $95 \%$ confidence interval and significance was $\mathrm{p}<0.05$.

\section{Results}

The mean age of the patients was $37.95 \pm 13.28$ years (range 19-71). Psychiatric disorders were major depression in 9 patients (39\%), catatonia in 2 (9\%), bipolar disorder in $8(34 \%)$, obsessive-compulsive disorder and comorbid depression in 2 (9\%) and schizophrenia in 2 (9\%). Patients did not receive any cardiotoxic antidepressant or other psychopharmacological agents, antidepressants that affect blood pressure such as venlafaxine and duloxetine were used for 7 patients (30.4\%) and polypharmacy was used for $18(78.3 \%)$. The mean seizure duration was $29.53 \pm 4.12 \mathrm{~s}$ (range $23-37$ ) and the total seizure duration was $206.72 \pm 27.93 \mathrm{~s}$ (range 172-243). There was no change in ejection fraction or heart rate before and after ECT. The detailed echocardiographic findings at baseline and after the 1st and 7th ECT are given in table 1. There was no statistical difference in the echocardiographic findings except for the A velocity. The echocardiographic A velocity values at baseline and after the 1 st and 7 th ECT were $63.8 \pm 14.1,68.4 \pm 13.3$ and $69.3 \pm 14.2 \mathrm{~cm} / \mathrm{s}$, respectively. Compared to the baseline, the differences were statistically significant: $\mathrm{p}=0.008$ (1st ECT) and $\mathrm{p}=$ 0.017 (7th ECT). There was no relationship between the increase of inflow A velocity and the type of pharmacotherapy (type of antidepressant or polypharmacy) and total and mean duration of the seizures.

\section{Discussion}

The main finding of our study was a consistently increased mitral inflow A wave velocity after recurrent ECT. ECT did not affect other echocardiographic parameters including the tissue Doppler parameters.

The curative mechanism of ECT is still unknown. The electrical current triggers widespread convulsions and increases cerebral blood flow and intracranial pressure [6]. Cardiovascular response is mediated mainly by increased 
Table 1. Echocardiographic findings at baseline and after the first and last ECT

\begin{tabular}{lllll}
\hline Echocardiographic variables & Baseline & After the first ECT & After the last ECT & p values \\
\hline E velocity, cm/s & $73.6 \pm 13.4$ & $76.2 \pm 17.5$ & $75.4 \pm 16.2$ & $0.161 ; 0.463$ \\
A velocity, cm/s & $63.8 \pm 14.1$ & $68.4 \pm 13.3$ & $69.3 \pm 14.2$ & $0.008 ; 0.017$ \\
E' velocity, cm/s & $14.1 \pm 3.6$ & $14.9 \pm 3.6$ & $13.4 \pm 2.8$ & $0.083 ; 0.310$ \\
E/A ratio & 1.22 & 1.16 & 1.1 & $0.216 ; 0.055$ \\
E/E' ratio & 5.41 & 5.27 & 5.61 & $0.4 ; 0.453$ \\
Ejection fraction, \% & 65 & 65 & 65 & $1 ; 1$ \\
Heart rate, min & $82.9 \pm 19.2$ & $83.2 \pm 17.7$ & $87.4 \pm 18.4$ & $0.760 ; 0.330$ \\
\hline
\end{tabular}

$\mathrm{A}=$ Late mitral inflow; $\mathrm{E}=$ early mitral inflow; E' = early diastolic mitral annulus motion; ECT = electroconvulsive therapy; $\mathrm{EF}=$ ejection fraction.

sympathetic tone, which comes after a sudden and short duration of increased parasympathetic tone. Sympathetic effects (shown on echocardiography) continue, on average, for $6 \mathrm{~h}$ after ECT. Wide fluctuations in autonomic tone are thought to be responsible for the arrhythmia and myocardial ischemia [2]. Patients with a prior history of cardiovascular disease, in particular, are at increased risk of such complications. Messina et al. [7] showed that ECT causes ischemic changes and segmental wall motion abnormalities seen on echocardiography in patients with cardiovascular disease or risk factors for atherosclerosis. In this study, the history of known cardiovascular diseases and atherosclerotic risk factors were excluded and segmental wall motion abnormalities were not detected. Increased sympathetic tonus associated with ECT provokes myocardial ischemia. Kadoi et al. [3], who followed patients closely with echocardiography throughout the ECT session, showed that the end-systolic diameter increased and that fractional shortening decreased. This decreased systolic performance is also associated with sympathetic hyperactivity after the ECT period. Fuenmayor et al. [8] reported that changes in the LV diameter and function were observed $20 \mathrm{~min}$ after the procedure but that these had returned to normal after $6 \mathrm{~h}$. In addition, they noted that the E/A ratio of mitral flow was reduced early after the procedure. However, in our study, there was no significant change in diastolic parameters, except that the mitral diastolic inflow A wave velocity increased 20 min after ECT. A probable explanation could be that, in this study, tissue Doppler was used, and this is a more accurate method. The increase in velocity persisted after recurrent ECT sessions. This finding might be considered as an indicator of acutely increased sympathetic tone.

Repetitive myocardial ischemia of short duration, also known as ischemic preconditioning, is the most important protective mechanism in myocardial infarction [9]. The question is whether or not the negative effects of ECT on the myocardium diminish after recurrent ECT. The situation is similar to that in ischemic preconditioning. A study on epileptic patients who had widespread convulsions comparable to ECT, revealed that the aortic elastic properties were impaired [10]. In addition, it has been shown that the recurrent sympathetic stimulation observed in epileptic patients triggers perivascular and interstitial fibrosis in the myocardium. Alehan et al. [11] revealed that CK-MB and BNP levels were increased $12 \mathrm{~h}$ after the seizures and these revealed subclinical myocardial injury.

In this study, there were some limitations, including a small sample size, patients who did not have the same diagnoses, the use of pharmacotherapy that was neither ideal for the patients nor free of cardiologic side effects, and the fact that the echocardiographic evaluation was made only before and $20 \mathrm{~min}$ after the 1st ECT and $20 \mathrm{~min}$ after the last ECT session.

\section{Conclusion}

The mitral diastolic inflow A wave velocity increased 20 min after ECT and this increase persisted after recurrent ECT sessions. This finding could be considered as an indicator of acutely increased sympathetic tone. Therefore, we recommend that ECT teams (the psychiatrists, anesthesiologists and nurses involved) should be aware of increased sympathetic tone and keep patients under observation after each ECT session for at least $20 \mathrm{~min}$. We therefore recommend that ECT units include a recovery room. There is a need for further studies with larger sample sizes to determine the ideal duration of the period for keeping patients under observation. 


\section{References}

1 Simpson KH, Lynch L: Anesthesia and electroconvulsive therapy (ECT). Anesthesia 1998;53:615-617.

$>2$ Ding Z, White PF: Anesthesia for electroconvulsive therapy. Anesth Analg 2002;94:13511364.

3 Kadoi Y, Saito S, Seki S, et al: Electroconvulsive therapy impairs systolic performance of the left ventricle. Can J Anaesth 2001;48:405408.

4 Cil H, Bulur S, Türker Y, et al: Impact of body mass index on left ventricular diastolic dysfunction. Echocardiography 2012;29:647651 .
Saraiva RM, Duarte DM, Duarte MP, et al: Tissue Doppler imaging identifies asymptomatic normotensive diabetics with diastolic dysfunction and reduced exercise tolerance. Echocardiography 2005;22:561-570.

6 Saito S, Miyoshi S, Yoshikawa D, et al: Regional cerebral oxygen saturation during electroconvulsive therapy: monitoring by nearinfrared spectrophotometry. Anesth Analg 1996;83:726-730.

7 Messina AG, Paranicas M, Katz B, et al: Effect of electroconvulsive therapy on the electrocardiogram and echocardiogram. Anesth Analg 1992;75:511-514.

$>8$ Fuenmayor AJ, el Fakih Y, Moreno J, et al: Effects of electroconvulsive therapy on cardiac function in patients without heart disease. Cardiology 1997;88:254-257.
9 Celik T, Celik M, Iyisoy A: Coronary collateral circulation (in Turkish). Turk Kardiyol Dern Ars 2010;38:505-514.

10 Ozmen N, Guney MS, Togrul E, et al: Elastic characteristics of aorta in patients with epilepsy. Med Glas (Zenica) 2011;88:15-18.

11 Alehan F, Erol I, Cemil T, et al: Elevated CK$\mathrm{MB}$ mass and plasma brain-type natriuretic peptide concentrations following convulsive seizures in children and adolescents: possible evidence of subtle cardiac dysfunction. Epilepsia 2009;50:755-760. 